\title{
Resolving the contributions of the membrane-bound and periplasmic nitrate reductase systems to nitric oxide and nitrous oxide production in Salmonella enterica serovar Typhimurium
}

\author{
Gary ROWLEY*1, Daniela HENSEN*, Heather FELGATE*, Anke ARKENBERG*, Corinne APPIA-AYME*, Karen PRIOR*, \\ Carl HARRINGTON $\dagger$, Sarah J. FIELD*, Julea N. BUTT* ${ }^{\star}$, Elizabeth BAGGS $\ddagger$ and David J. RICHARDSON*1 \\ *School of Biological Sciences, University of East Anglia, Norwich Research Park, Norwich NR4 7TJ, U.K., †'Institute of Food Research, Norwich Research Park, Colney, Norwich \\ NR4 7UA, U.K., and łInstitute of Biological and Environmental Sciences, University of Aberdeen, St Machar Drive, Aberdeen AB24 3UU, U.K.
}

\begin{abstract}
The production of cytotoxic nitric oxide (NO) and conversion into the neuropharmacological agent and potent greenhouse gas nitrous oxide $\left(\mathrm{N}_{2} \mathrm{O}\right)$ is linked with anoxic nitrate catabolism by Salmonella enterica serovar Typhimurium. Salmonella can synthesize two types of nitrate reductase: a membrane-bound form (Nar) and a periplasmic form (Nap). Nitrate catabolism was studied under nitrate-rich and nitrate-limited conditions in chemostat cultures following transition from oxic to anoxic conditions. Intracellular NO production was reported qualitatively by assessing transcription of the NO-regulated genes encoding flavohaemoglobin (Hmp), flavorubredoxin (NorV) and hybrid cluster protein (Hcp). A more quantitative analysis of the extent of $\mathrm{NO}$ formation was gained by measuring production of $\mathrm{N}_{2} \mathrm{O}$, the end-product of anoxic NO-detoxification. Under nitrate-rich conditions, the nar, nap, hmp, norV and hcp genes were all induced following transition from the oxic to anoxic state, and
\end{abstract}

$20 \%$ of nitrate consumed in steady-state was released as $\mathrm{N}_{2} \mathrm{O}$ when nitrite had accumulated to millimolar levels. The kinetics of nitrate consumption, nitrite accumulation and $\mathrm{N}_{2} \mathrm{O}$ production were similar to those of wild-type in nitrate-sufficient cultures of a nap mutant. In contrast, in a narG mutant, the steady-state rate of $\mathrm{N}_{2} \mathrm{O}$ production was $\sim 30$-fold lower than that of the wildtype. Under nitrate-limited conditions, nap, but not nar, was upregulated following transition from oxic to anoxic metabolism and very little $\mathrm{N}_{2} \mathrm{O}$ production was observed. Thus a combination of nitrate-sufficiency, nitrite accumulation and an active Nar-type nitrate reductase leads to $\mathrm{NO}$ and thence $\mathrm{N}_{2} \mathrm{O}$ production, and this can account for up to $20 \%$ of the nitrate catabolized.

Key words: Enterobacteriaceae, nitrate reductase, nitric oxide, nitrite reductase, nitrous oxide, Salmonella.

\section{INTRODUCTION}

The Enterobacteriaceae family of Gammaproteobacteria are found naturally in soils, water systems and sewage and as a part of the gut flora in the gastrointestinal tract. They are facultative anaerobes that can, with few exceptions, use nitrate or nitrite as terminal respiratory electron acceptors. The availability of these electron acceptors varies in the different environments to which the bacteria adapt [1]. For an enteric pathogen, for example, the gastrointestinal tract can be rich in nitrate or nitrite, but nitrate is more scarce in bodily fluids such as the bloodstream [1]. In many species of Enterobacteriaceae, there are two biochemically distinct nitrate reductases: one membrane-bound with the active site located in the cytoplasm and the other in the periplasm. These are coupled to two nitrite reductases to provide parallel pathways for nitrate reduction to ammonium in the two cellular compartments and that are differentially expressed in response to different nitrate and nitrite concentrations $[1,2]$. In the cytoplasm, nitrate is reduced to nitrite by a membrane-bound respiratory nitrate reductase system (NarGHI):

$\mathrm{NO}_{3}^{-}+2 \mathrm{e}^{-}+2 \mathrm{H}^{+} \rightarrow \mathrm{NO}_{2}^{-}+\mathrm{H}_{2} \mathrm{O}$ (Reaction 1).

The nitrite produced can then be reduced further to ammonium by a sirohaem-containing nitrite reductase (NirB):
$\mathrm{NO}_{2}^{-}+6 \mathrm{e}^{-}+8 \mathrm{H}^{+} \rightarrow \mathrm{NH}_{4}^{+}+2 \mathrm{H}_{2} \mathrm{O}$ (Reaction 2).

In the periplasm, the process involves two different enzymes: a periplasmic nitrate reductase (NapA) that reduces nitrate to nitrite and a periplasmic cytochrome $c$ nitrite reductase (NrfA) that further reduces the nitrite to ammonium. Reactions 1 and 2 together lead to the production of extracellular ammonium and are often termed DNRA (dissimilatory nitrate reduction to ammonium).

Salmonella and Escherichia coli produce the cytotoxin nitric oxide (NO) as a side-product of nitrate or nitrite metabolism $[3,4]$. One major source of this NO has been suggested to be the reduction of nitrite by the membrane-bound nitrate reductase NarG [3]. This endogenous NO leads to derepression of genes encoding systems that are concerned with the detoxification of $\mathrm{NO}$ and the repair of proteins damaged by the cytotoxin. The regulator that mediates this derepression is the NO-binding protein NsrR (NO-sensing repressor) [5-7]. A key enzyme in the NsrR regulon in Salmonella enterica serovar Typhimurium is flavohaemoglobin (Hmp) that reduces two molecules of NO to one molecule of nitrous oxide $\left(\mathrm{N}_{2} \mathrm{O}\right)$ under anoxic conditions, using cytoplasmic NADH as electron donor $[8,9]$. This represents the conversion of a potent cytotoxin into a product that is both a neuropharmacological agent and potent greenhouse gas [10]. There have been reports of $\mathrm{N}_{2} \mathrm{O}$ release by pure cultures

Abbreviations used: D, dilution rate; DNRA, dissimilatory nitrate reduction to ammonium; Hmp, flavohaemoglobin; Km ${ }^{R}$, kanamycin-resistance; MS, minimal salts; NapA, periplasmic nitrate reductase; Nar, membrane-bound nitrate reductase; NirB, cytoplasmic sirohaem nitrite reductase; NorV, flavorubredoxin; NrfA, periplasmic cytochrome $c$ nitrite reductase; NsrR, NO-sensing repressor; qc, specific rate of consumption; qRT, quantitative real-time; $\mathrm{S}_{\mathrm{BR}}$, bioreactor concentration of substrate; $\mathrm{S}_{\mathrm{R}}$, reservoir concentration.

Correspondence may be addressed to either of these authors (email g.rowley@uea.ac.uk or d.richardson@uea.ac.uk). 
Table 1 All strains and plasmids used in the present study

\begin{tabular}{|c|c|c|}
\hline Strain or plasmid & Genotypes or relevant characteristics & Reference \\
\hline \multicolumn{3}{|l|}{ Strains } \\
\hline SL1344 & Salmonella Typhimurium, his- ${ }^{-}$, mouse-virulent & {$[40]$} \\
\hline$\triangle h m p A$ & SL1344 $\Delta$ hmpA::kan & [29] \\
\hline$\Delta$ norV & SL1344 $\Delta$ norV::cat & [29] \\
\hline$\Delta$ norV $\Delta h m p A$ & SL1344 $\Delta h m p A::$ kan $\Delta$ norV:::cat & [29] \\
\hline$\Delta$ narGHJl & SL1344 $\Delta$ narGHJl::kan & The present study \\
\hline$\triangle$ napFDAGHBC & SL1344 $\triangle$ napFDAGHBC::kan & The present study \\
\hline$\Delta$ nirB & SL1344 $\Delta$ nirB::kan & The present study \\
\hline$\Delta h c p$ & Sl1344 $\Delta$ hcp::kan & The present study \\
\hline \multicolumn{3}{|l|}{ Plasmids } \\
\hline pKD4 & $A p^{R}, p A N T-S \gamma$ derivative containing an FRT-flanked $\mathrm{Km}^{R}$ & {$[17]$} \\
\hline pKD46 & $A p^{R}$, plNT-ts derivative containing araC- $\mathrm{P}_{\text {arab }}$ and $\gamma, \beta$, exo genes & [17] \\
\hline
\end{tabular}

of Enterobacteriaceae, including Escherichia coli, Klebsiella pneumoniae and S. enterica during nitrate metabolism, and it is likely that this is due to reductive detoxification of NO produced as a side-product of nitrate metabolism [11,12,18-20]. In the present paper, we describe a biochemical study on nitrate catabolism in continuous cultures of the food-borne pathogen Salmonella Typhimurium which reveals that intracellular NO production and associated extracellular $\mathrm{N}_{2} \mathrm{O}$ production can account for up to $20 \%$ of nitrate catabolized and is linked to the culture nitrate status, nitrite accumulation from nitrate respiration and the biochemical type of the nitrate reductase system expressed.

\section{EXPERIMENTAL}

\section{Bacterial strains and growth media}

Salmonella Typhimurium strains (Table 1) were cultivated anaerobically in MS (minimal salts) medium [13]. The sole carbon and electron source was glycerol, the sole terminal electron acceptor was sodium nitrate, with ammonium $(15 \mathrm{mM})$ present as a nitrogen source. Continuous culture was performed in a New Brunswick Scientific BioFlo 3000 fermenter with a 1.2 litre working volume under $\mathrm{pH}$ control $(\mathrm{pH} 7.0,1 \mathrm{M} \mathrm{NaOH}$ and $1 \mathrm{M}$ $\mathrm{HCl} / 0.1 \mathrm{M} \mathrm{H}_{2} \mathrm{SO}_{4}$ used for regulation). A $100 \mathrm{ml}$ volume of MS medium was inoculated with $5 \mathrm{ml}$ of an overnight culture and aerobically incubated overnight at $37^{\circ} \mathrm{C}$. Then, $50 \mathrm{ml}$ of this culture was used to inoculate the bioreactor. After $24 \mathrm{~h}$ of aerobic batch growth, the air supply was switched off and a feed of MS medium was started to achieve a dilution rate (D) of $0.04 \mathrm{~h}^{-1}$. The measured dissolved $\mathrm{O}_{2}$ (percentage air saturation) in the culture fell from $100 \%$ to $0 \%$ within $1 \mathrm{~h}$ of switching off the air supply and was monitored throughout the continuous culture phase of the experiment to ensure that it remained at $0 \%$. During the experiment, samples were taken at regular intervals to determine the attenuance at $600 \mathrm{~nm}\left(D_{600}\right)$, protein concentration and nitrogen compound composition.

\section{Protein and nitrogen compound quantification}

Protein concentration was determined using the method described in [14]. Nitrate was determined via HPLC using the anionexchange column Ion Pac AS22, $2 \mathrm{~mm} \times 250 \mathrm{~mm}$ (Dionex, ICS900) as described by the manufacturer. Nitrite was measured colorimetrically with a modified Griess reaction [15]. A PerkinElmer Clarus ${ }^{\circledR} 500$ Gas Chromatograph with an ECD (Electron Capture Detector) and Elite-PLOT Q (DVB Plot Column, $30 \mathrm{~m}$ length; $0.53 \mathrm{~mm}$ internal diameter; carrier gas, $\mathrm{N}_{2}$; make-up gas, $95 \%$ argon $/ 5 \%$ methane; temperatures as described by the manufacturer) were used with known gas standards of $\mathrm{N}_{2} \mathrm{O}$ [0.4, 100, 1000 p.p.m., supplied from StGas] to determine the $\mathrm{N}_{2} \mathrm{O}$ concentration in headspace gas samples. From this, the total $\mathrm{N}_{2} \mathrm{O}$ in headspace and solution was calculated by applying Henry's Law, assuming equilibration between the solution and gas phases and using a Henry's Law constant at $37^{\circ} \mathrm{C}$ of 0.453 .

\section{Enzyme activity}

The NarGH nitrate reductase complex, comprising the 140000 Da NarG and the 60000 Da NarH subunits, was purified from anaerobic cultures of Salmonella Typhimurium and assayed using Methyl Viologen as the electron donor, essentially as described previously for Paracoccus pantotrophus NarGH [16].

\section{RNA extraction, qRT (quantitative real-time)-PCR and mutant construction}

RNA was extracted at the appropriate time points from nitrate-sufficient and -limited continuous cultures of Salmonella Typhimurium SL1344 using a Promega SV 96 total RNA purification kit. The total RNAs were first treated with Turbo DNaseFree from Ambion and the absence of DNA contamination was verified by PCR. RNA quality was assessed on an Agilent 2100 Bioanalyser. Then, $2 \mu \mathrm{g}$ of DNaseI-treated total RNA were retro-transcribed from random hexamers (Invitrogen) with Superscript II RT (Invitrogen) according to the manufacturer's recommendations. Specific primers for the genes of interest amplifying an average product of $100 \mathrm{bp}$ with an approximate $T_{\mathrm{m}}$ (melting temperature) of $60^{\circ} \mathrm{C}$ were designed. The qRTPCRs were performed on a 5-fold dilution of the total cDNA obtained, using the Bio-Rad Laboratories CFX96 instrument and SensiMix ${ }^{\mathrm{TM}}$ SYBR No-ROX kit (Bioline). The qRT-PCR experiments were performed in triplicate, with three independent total RNA preparations. The calculated threshold cycle $\left(C_{t}\right)$ for each gene was normalized to the $C_{t}$ of the $a m p D$ gene, expression of which is invariant across a large range of growth conditions. Mutants were constructed in Salmonella Typhimurium SL1344 using $\lambda$ Red mutagenesis [17]. Oligonucleotides were designed which deleted the entire gene or operon in question inclusive of start and stop codons. A linear PCR product was generated using a template plasmid, pKD4, resulting in a $\mathrm{Km}^{\mathrm{R}}$ (kanamycinresistance) gene cassette with $40 \mathrm{bp}$ of homologous sequence flanking to the loci. The amplified DNA fragment was column-purified and electroporated into Salmonella Typhimurium SL1344pKD46. Mutations were confirmed by PCR using primers external to the site of mutagenesis; as well as internal 

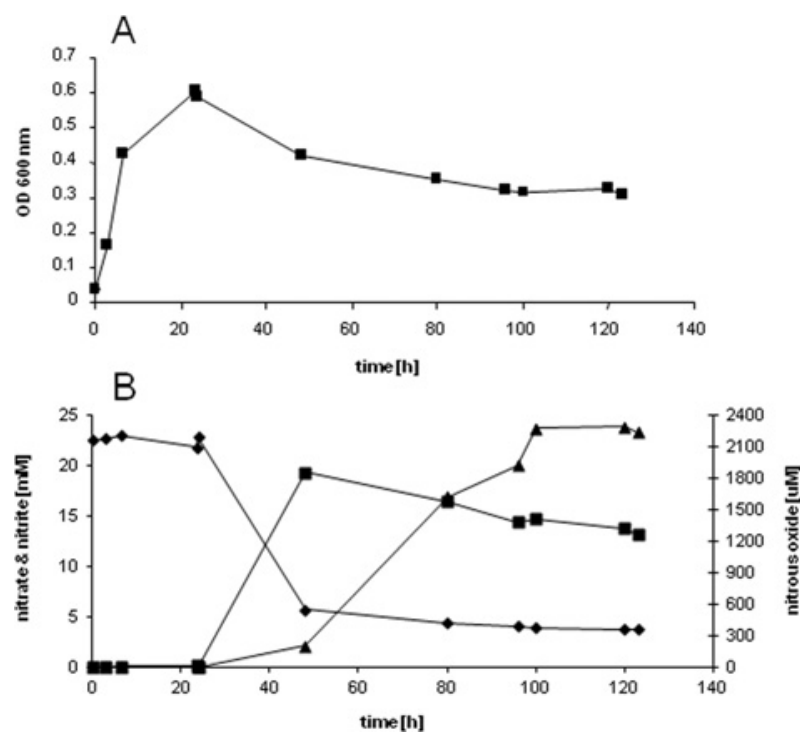

Figure 1 Nitrate, nitrite and $\mathrm{N}_{2} \mathrm{O}$ consumption or production in a glycerollimited nitrate-sufficient continuous culture of Salmonella Typhimurium SL1344

The culture was initially grown in batch mode under atmospheric oxygen concentration for $24 \mathrm{~h}$, during which time cellular biomass [indicated by an increase in $D_{600}$ ('OD')] was generated. The air supply to the culture was then switched off and the system switched to continuous mode at a dilution rate of $0.04 \mathrm{~h}^{-1}$. The measured dissolved $\mathrm{O}_{2}$ (percentage air saturation) in the culture fell from $100 \%$ to $0 \%$ within $1 \mathrm{~h}$ of switching off the air supply, and was monitored throughout the experiment to ensure it remained at $0 \%$. The $\mathrm{pH}$ and temperature were maintained at 7 and $37^{\circ} \mathrm{C}$ respectively. The glycerol concentration in the reservoir feed was $5 \mathrm{mM}$ and the nitrate concentration was $22.5 \mathrm{mM}$. (A) Biomass; (B) nitrate $(\boldsymbol{\bullet})$, nitrite ( $(\mathbf{\square})$ and $\mathrm{N}_{2} \mathrm{O}(\boldsymbol{\mathbf { \Delta }})$. For clarity, only the results from a single chemostat run are shown. The results $(\underline{\underline{S} \text { S.D. }}$ ) derived from replicate experiments is given in Table 2 .

primers within the $\mathrm{Km}^{\mathrm{R}}$ cassette, $\mathrm{k} 1$ and $\mathrm{k} 2$ [17]. $\mathrm{P} 22$ transduction was used to transfer the mutations into a clean SL1344 background.

\section{RESULTS}

\section{Nitrate respiration and $\mathrm{N}_{2} \mathrm{O}$ production in nitrate-sufficient continuous cultures}

Salmonella Typhimurium was cultured to anoxic steady-state in continuous cultures with nitrate (reservoir concentration, $S_{R}$, $22.5 \mathrm{mM}$ ) present as the respiratory electron acceptor and glycerol $\left(\mathrm{S}_{\mathrm{R}} 5 \mathrm{mM}\right)$ present as the carbon source for anabolism and electron source for respiration. The cultures were grown in batch mode under an atmospheric oxygen concentration for $24 \mathrm{~h}$, during which time cellular biomass (x) was generated (Figure 1A). The air supply to the culture was then switched off and the system was switched to continuous mode $\left(\mathrm{D}=0.04 \mathrm{~h}^{-1}\right)$. There was a decrease in $D_{600}$ and protein concentration in the bioreactor as the culture shifted from aerobic to anaerobic metabolism, until a new biomass steady-state was reached after three or four bioreactor vessel volume changes $(\sim 80-120 \mathrm{~h})$. During the transitional, non-steady-state, phase (between 24 and $\sim 80 \mathrm{~h}$ ) the nitrate concentration in the bioreactor $\left(\mathrm{S}_{\mathrm{BR}} \mathrm{NO}_{3}{ }^{-}\right)$decreased, consistent with a shift from oxygen respiration to anaerobic nitrate respiration (Figure 1B). The shift to nitrate respiration was also reflected by increased transcription of both the $n a r G$ and napA nitrate reductase genes and the gene for the nitrate/nitrite antiporter $n a r K$, detected using qRT-PCR analysis, at $80 \mathrm{~h}$ and $120 \mathrm{~h}$ compared with $5 \mathrm{~h}$ (Figure 2).
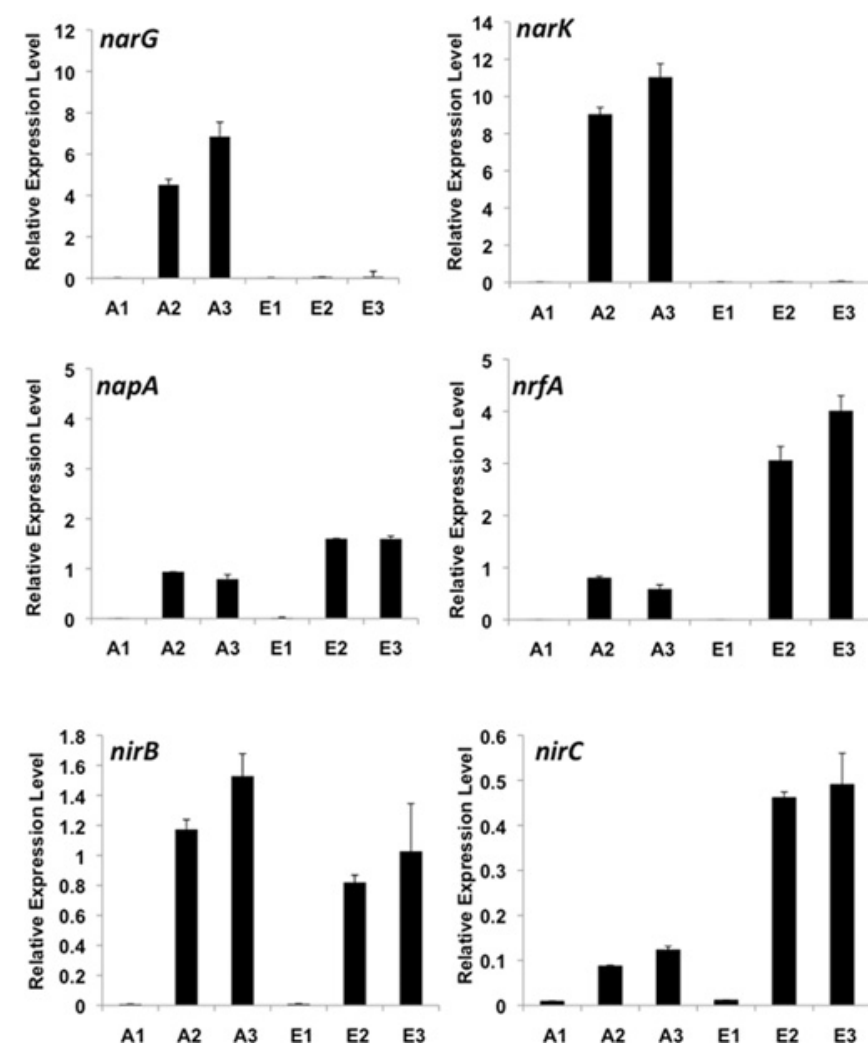

Figure 2 qRT-PCR of genes involved in nitrate and nitrite transport and reduction

The GRT-PCR experiments were performed in triplicate, with three independent total RNA preparations. The calculated threshold cycle $\left(C_{t}\right)$ for each gene was normalized to the $C_{t}$ of the ampD control. Results are means \pm S.D. A, nitrate sufficiency; E, nitrate limitation; 1,5 h oxic; 2, $80 \mathrm{~h}$ anoxic; $3,120 \mathrm{~h}$ anoxic.

Nitrite was produced almost stoichiometrically with nitrate consumption during the first $\sim 20 \mathrm{~h}$ of the transition phase (24-48 h) (Figure 1B). This is consistent with nitrite being produced directly from nitrate reduction by the nitrate reductase(s) (Reaction 1), such that the $\mathrm{S}_{\mathrm{BR}} \mathrm{NO}_{3}{ }^{-}$decreased from $\sim 22 \mathrm{mM}$ to $\sim 5 \mathrm{mM}$ and the $\mathrm{S}_{\mathrm{BR}} \mathrm{NO}_{2}{ }^{-}$increased from $\sim 0 \mathrm{mM}$ to $\sim 18 \mathrm{mM}$ (Figure 1B). Over the next $50 \mathrm{~h}$, the $\mathrm{S}_{\mathrm{BR}} \mathrm{NO}_{2}{ }^{-}$ decreased by $\sim 4.5 \mathrm{mM}$ from $\sim 18 \mathrm{mM}$ to a steady-state value of $\sim 14 \mathrm{mM}(100-120 \mathrm{~h})$. In steady state, the specific rate of nitrate consumption $\left(\mathrm{qcNO}_{3}{ }^{-}\right)$was $\sim 20 \%$ higher than the specific rate of nitrite accumulation ( $\mathrm{qpNO}_{2}{ }^{-}$) (Table 2). To account for this difference some of the nitrite produced from nitrate in Reaction 1 must be further consumed by the culture. This rate of consumption $\left(\mathrm{qcNO}_{2}{ }^{-}\right)$can be estimated from the difference between $\mathrm{qcNO}_{3}{ }^{-}$ and $\mathrm{qpNO}_{2}{ }^{-}$(Table 2). Ammonium is a possible net product of nitrite reduction by the NirB or NrfA nitrite reductases (Reaction 2), with nirB in particular being strongly induced during the anaerobic phase (Figure 2). However, the $\mathrm{S}_{\mathrm{BR}} \mathrm{NH}_{4}{ }^{+}$remained constant at $\sim 13 \mathrm{mM}$ throughout the continuous culture phase of the experiment, which represented a net consumption of $\sim 2 \mathrm{mM}$ of the $15 \mathrm{mM} \mathrm{NH}_{4}{ }^{+}$in the reservoir feed for anabolic purposes.

$\mathrm{NO}$ is also a potential net product of nitrite reduction, but extracellular accumulation was not detected above $1 \mu \mathrm{M}$. However, increased transcription of $h m p, h c p$ and norV was observed following the transition from oxic ( $5 \mathrm{~h}$ sampling time) to anoxic ( 80 and $100 \mathrm{~h}$ sampling times) conditions (Figure 3 ). These genes are regulated by the cytoplasmic NO-responsive transcription factors NsrR ( $h m p$ and $h c p$ ) and NorR (norV), 
Table 2 The steady-state rates of nitrate, nitrite and $\mathrm{N}_{2} \mathrm{O}$ production in chemostat cultures of Salmonella Typhimurium

The data presented are taken during steady state at $100-120 \mathrm{~h}$ from replicate continuous cultures run in parallel under identical conditions. $\mathrm{qCNO}_{3}{ }^{-}=\left(\mathrm{S}_{\mathrm{R}} \mathrm{NO}_{3}{ }^{-}-\mathrm{S}_{\mathrm{BR}} \mathrm{NO}_{3}{ }^{-}\right) \mathrm{D} / \mathrm{x}_{\text {; }}$ $\mathrm{qpNO}_{2}{ }^{-}=\left(\mathrm{S}_{\mathrm{BR}} \mathrm{NO}_{2}{ }^{-}\right) \mathrm{D} / \mathrm{x} ; \mathrm{qCNO}_{2}{ }^{-}=\mathrm{qCNO}_{3}{ }^{-}-\mathrm{qpNO}_{2}{ }^{-} ; \mathrm{qpN}_{2} \mathrm{O}=\left(\mathrm{S}_{\mathrm{BR}} \mathrm{N}_{2} \mathrm{O}\right) \mathrm{D} / \mathrm{x} . \mathrm{N}_{2} \mathrm{O}$ data are expressed as nitrogen-equivalents to allow for direct comparison between $\mathrm{N}_{2} \mathrm{O}$ and nitrate or nitrite. ND, not detectable, nitrite detection limit $=0.005 \mathrm{mM}$.

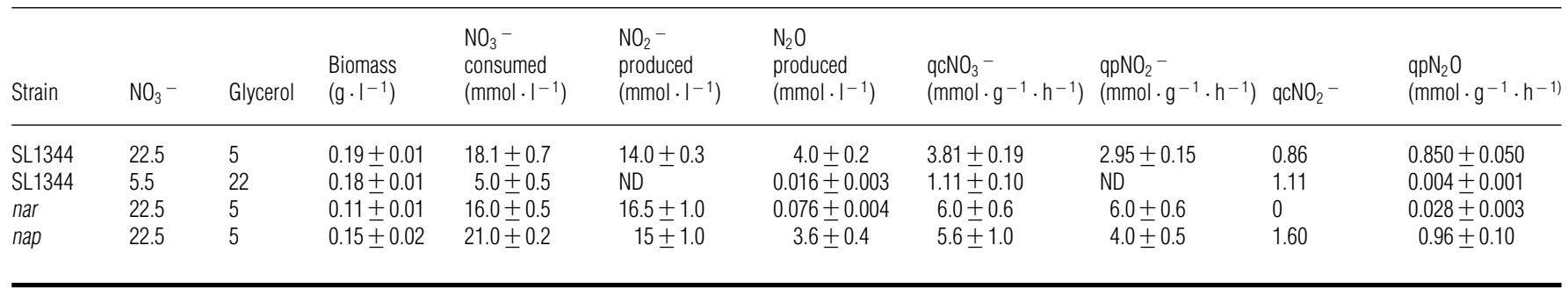
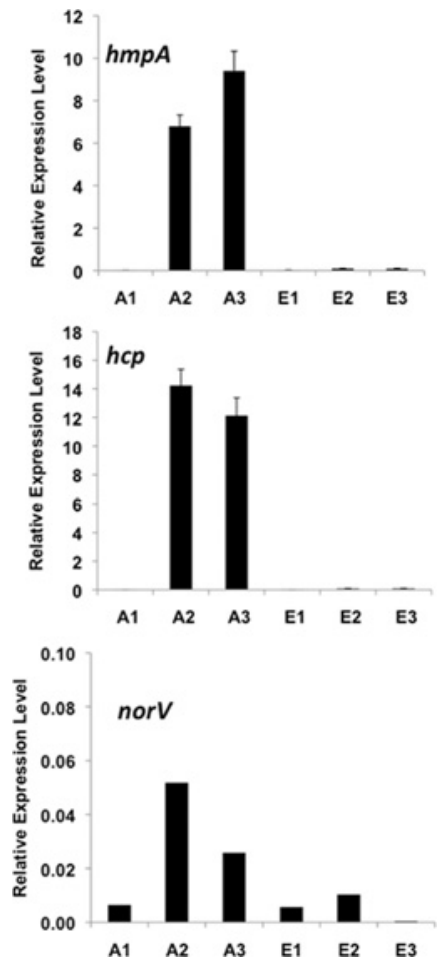

Figure 3 qRT-PCR of genes regulated by the NO-responsive transcription factors NsrR and NorR

The qRT-PCR experiments were performed in triplicate, with three independent total RNA preparations. The calculated threshold cycle $\left(C_{t}\right)$ for each gene was normalized to the $C_{t}$ of the ampD control. Results are means \pm S.D. A, nitrate sufficiency; $E$, nitrate limitation; $1,5 \mathrm{~h}$ oxic; $2,80 \mathrm{~h}$ anoxic; $3,120 \mathrm{~h}$ anoxic.

and so this increased transcription is indicative of intracellular $\mathrm{NO}$ production. It was notable that $\mathrm{N}_{2} \mathrm{O}$ accumulated as the $\mathrm{S}_{\mathrm{BR}} \mathrm{NO}_{2}{ }^{-}$decreased in the transition phase between 48 and $100 \mathrm{~h}$ (Figure 1B). The steady-state rate of $\mathrm{N}_{2} \mathrm{O}$ production $\left(\mathrm{qpN}_{2} \mathrm{O}\right)$, when normalized for the two nitrogens in $\mathrm{N}_{2} \mathrm{O}$ compared with the one nitrogen in $\mathrm{NO}_{2}{ }^{-}$, matched the steady-state rate of nitrite consumption (Table 2), showing that this $\mathrm{N}_{2} \mathrm{O}$ production is closely linked to the metabolism of nitrite generated from nitrate metabolism. Since $h m p, h c p$ and norV transcription indicated the production of intracellular $\mathrm{NO}$, then the series of reactions that lead to $2 \mathrm{~mol}$ of $\mathrm{NO}_{2}{ }^{-}$being reduced to $1 \mathrm{~mol}$ of $\mathrm{N}_{2} \mathrm{O}$ via $\mathrm{NO}$ is predicted to be:

$2\left[\mathrm{NO}_{2}{ }^{-}+2 \mathrm{H}^{+}+\mathrm{e}^{-} \rightarrow \mathrm{NO}+\mathrm{H}_{2} \mathrm{O}\right]($ Reaction 3$)$
$2 \mathrm{NO}+2 \mathrm{H}^{+}+2 \mathrm{e}^{-} \rightarrow \mathrm{N}_{2} \mathrm{O}+\mathrm{H}_{2} \mathrm{O}$ (Reaction 4$)$

Reaction 4 is known to be catalysed by both the Hmp and NorV (flavorubredoxin) enzymes under anoxic conditions, and so hmp and norV mutants were also analysed under the nitrate-sufficient continuous culture conditions. In the case of strains carrying single lesions in either $h m p$ or $h c p$, the rate of $\mathrm{N}_{2} \mathrm{O}$ production in steady state was comparable with wild-type. However, a double hmp nor mutant only produced $\mathrm{N}_{2} \mathrm{O}$ at $\sim 40 \%$ of the rate of the wild-type, suggesting functional overlap of these two systems in $\mathrm{NO}$ detoxification and $\mathrm{N}_{2} \mathrm{O}$ production under anoxic conditions. In total, $\sim 18 \mathrm{mM}$ nitrate in the feed reservoir was consumed in the steady state $\left(\mathrm{S}_{\mathrm{R}} \mathrm{NO}_{3}{ }^{-}-\mathrm{S}_{\mathrm{BR}} \mathrm{NO}_{3}{ }^{-}\right.$at $\left.t=120 \mathrm{~h}\right)$ and $\sim 4 \mathrm{mM}$ nitrogen equivalents of $\mathrm{N}_{2} \mathrm{O}$ was produced (Table 2). This represents a conversion of $\sim 20 \%$ of nitrate into $\mathrm{N}_{2} \mathrm{O}$ (Table 2).

\section{Comparison of $\mathrm{N}_{2} \mathrm{O}$ production in Salmonella Typhimurium nar and nap mutants in nitrate-sufficient continuous cultures}

The NarG nitrate reductase has previously been implicated in both $\mathrm{NO}$ and $\mathrm{N}_{2} \mathrm{O}$ production and nitrosation in Enterobacteriaceae $[2,3,11,12,18-20]$. However, a number of Enterobacteriaceae species only have a Nap type of nitrate reductase [2], and, in those that have both Nar and Nap, the nitrate-rich growth conditions under which many previous studies of $\mathrm{NO}$ or $\mathrm{N}_{2} \mathrm{O}$ production has been made would not be those that favour nap expression, which is maximal under nitrate-limiting conditions [1,2,5,21-23]. Thus the question of whether activity of Nap can lead to NO and $\mathrm{N}_{2} \mathrm{O}$ production has not been directly addressed before. To investigate this, isogenic narG and napA strains were constructed. Both were able to grow under anaerobic conditions with nitrate as a sole electron acceptor, but a double narG napA mutant could not. Under the nitrate-rich continuous culture conditions, the nar strain, which is dependent on Nap for growth, achieved a steady-state anoxic biomass of $\sim 60 \%$ of the wild-type strain under identical culture conditions (Figure 4A and Table 2). The kinetics of nitrate consumption and nitrite accumulation during the aerobic-anaerobic transition phase were closely matched (Figure 4B and Table 2), suggesting that the respiratory nitrite reductase systems Nrf or Nir (Reaction 2) do not operate at a significant level to consume the nitrite produced from Reaction 1. The rate of nitrate consumption in the steady state (100-120 h) was comparable with that of the wild-type (Table 2). Significantly, however, the steady-state $\mathrm{S}_{\mathrm{BR}} \mathrm{N}_{2} \mathrm{O}$ was only $\sim 0.08 \mathrm{mM}$ in the nar strain (Figure 4B and Table 2), such that the steady-state rate of $\mathrm{N}_{2} \mathrm{O}$ production $\left(\mathrm{qpN}_{2} \mathrm{O}\right)$ was approximately 30 -fold lower than for the wild-type strain (Table 2). In contrast, the rate of $\mathrm{N}_{2} \mathrm{O}$ production in the nap strain was comparable with that of 

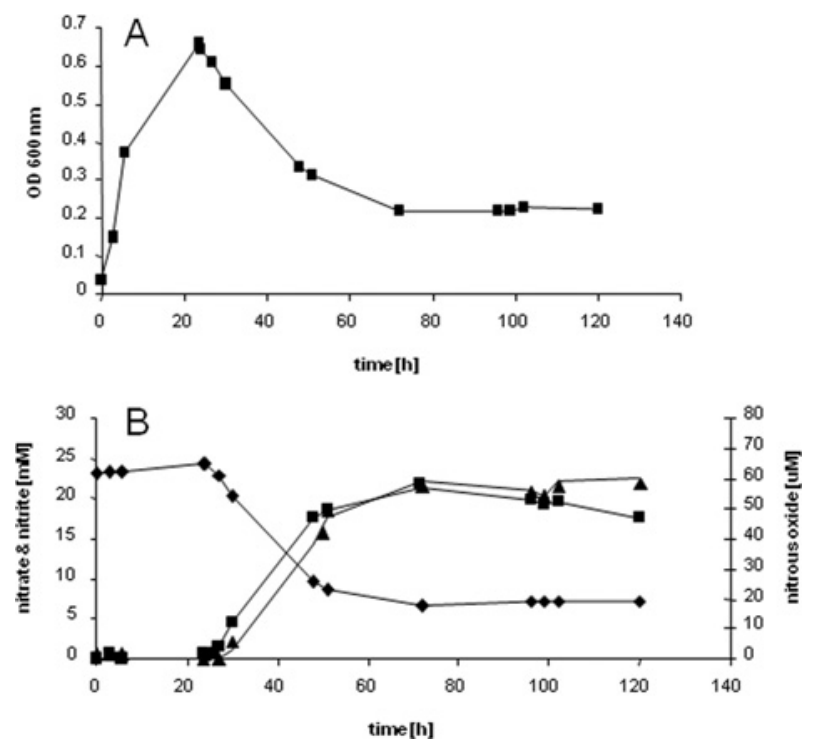

Figure 4 Nitrate, nitrite and $\mathrm{N}_{2} \mathrm{O}$ consumption or production in a glycerollimited nitrate-sufficient continuous culture of an Salmonella Typhimurium mutant deficient in Nar

The culture was run as described for Figure 1. The glycerol concentration in the reservoir feed was $5 \mathrm{mM}$ and nitrate concentration was $22 \mathrm{mM}$. (A) Biomass; (B) nitrate ( $)$, nitrite (ם) and $\mathrm{N}_{2} \mathrm{O}(\boldsymbol{\Delta})$. For clarity, only the results from a single chemostat run are shown. The results $( \pm$ S.D.) derived from replicate experiments is given in Table 2 .

the wild-type (Table 2). The large difference in the rate of $\mathrm{N}_{2} \mathrm{O}$ production between the nap and nar strains (Table 2) suggests that it is not associated with respiration in nitrate-rich cultures conditions itself, but with metabolism via the Nar system, rather than the Nap system, under nitrate-rich culture conditions.

NarG generates nitrite in the cytoplasm and it has been argued, on the basis of genetic and microbiological data, that this can compete with nitrate for the Nar active site in the cytoplasm [3], with Nar then catalysing Reaction 3. To demonstrate biochemically that purified Salmonella Nar can reduce nitrite, we isolated the membrane-associated NarGH complex from detergent-solubilized membranes of Salmonella Typhimurium. The enzyme complex displayed nitrate and nitrite reductase activities that each obeyed a Michaelis-Menten-type dependency on substrate concentration (Figure 5). These activities were fully sensitive to low concentrations of azide $(20 \mu \mathrm{M})$, which is a potent inhibitor of Nar-type nitrate reductases and NO production and nitrosation by Enterobacteriacaea $[19,24]$. The $K_{\mathrm{m}}$ and $V_{\max }$ values for nitrate reduction were determined as $123 \pm 14 \mu \mathrm{M}$ and $83 \pm 10 \mu \mathrm{mol} \cdot \mathrm{min}^{-1} \cdot \mathrm{mg}^{-1}$ respectively (Figure $5 \mathrm{~A}$ ). For nitrite reduction, the $K_{\mathrm{m}}$ and $V_{\max }$ values were determined as $5200 \pm 1900 \mu \mathrm{M}$ and $24 \pm 5 \mu \mathrm{mol} \cdot \mathrm{min}^{-1} \cdot \mathrm{mg}^{-1}$ respectively (Figure 5B). Using a molecular mass for NarGH of $200000 \mathrm{Da}$, the $k_{\text {cat }}$ values for nitrate and nitrite could be approximated as 270 $\mathrm{s}^{-1}$ and $80 \mathrm{~s}^{-1}$ respectively.

In addition to Nar reducing nitrite to NO, it is possible that nitrite generated in the cytoplasm by Nar is a substrate for another NO-generating nitrite-reducing enzyme. The likely candidate for this would be the cytoplasmic NADH-dependent nitrite reductase NirB, which was also induced following transition to anoxic steady state (Figure 2), and has been implicated previously in cytoplasmic NO generation [25]. A nirB strain was therefore constructed and grown in the nitrate-rich continuous cultures, but the behaviour was identical with that of wild-type, with the specific rate of $\mathrm{N}_{2} \mathrm{O}$ production was similar to that of wild-type.
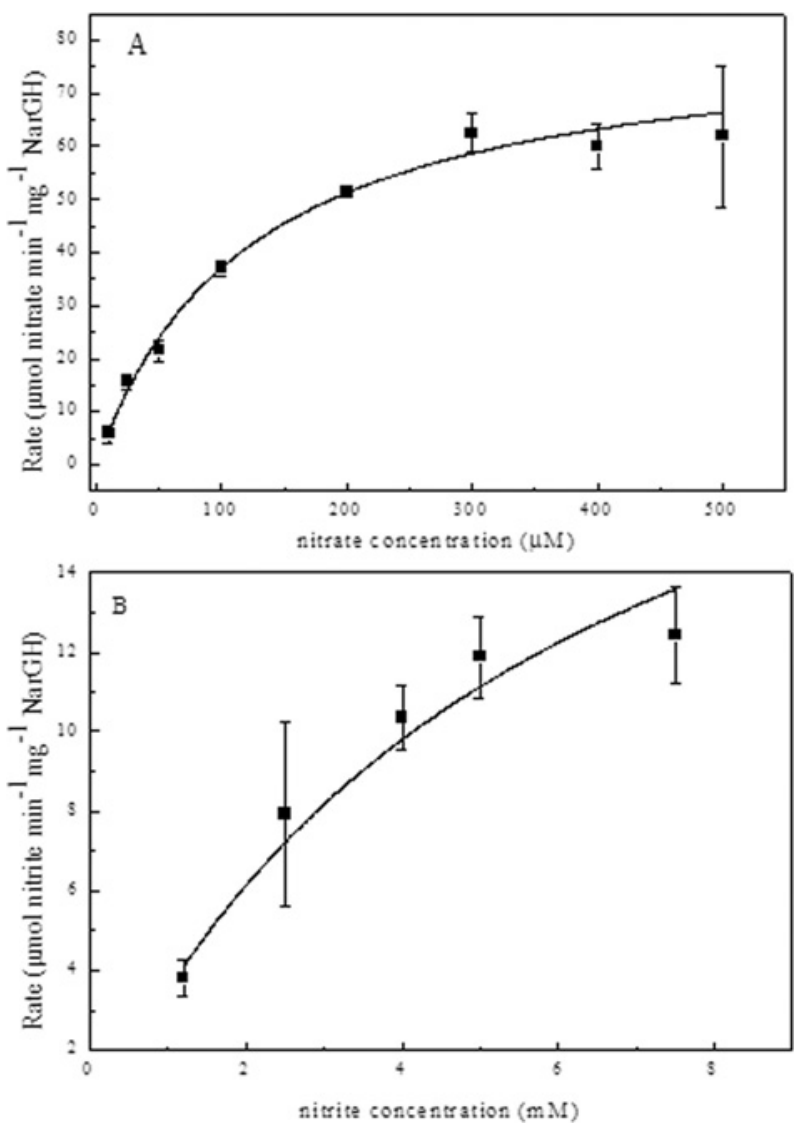

Figure 5 The kinetics of nitrate (A) nitrite (B) reduction by NarGH from Salmonella Typhimurium

Assays were undertaken in $20 \mathrm{mM}$ Hepes and $2 \mathrm{mM}$ EDTA (pH 7.0) under anaerobic conditions in nitrogen-sparged sealed cuvettes using reduced Methyl Viologen as electron donor. The data are fitted to the Michaelis-Menten kinetic model with the $K_{\mathrm{m}}$ and $V_{\text {max }}$ values for nitrate reduction as $123 \pm 14 \mu \mathrm{M}$ and $82 \pm 10 \mu \mathrm{mol} \cdot \mathrm{min}^{-1} \cdot \mathrm{mg}^{-1}$ respectively, and $K_{\mathrm{m}}$ and $V_{\text {max }}$ values for nitrite reduction as $5200 \pm 1900 \mu \mathrm{M}$ and $24 \pm 5 \mu \mathrm{mol} \cdot \mathrm{min}^{-1} \cdot \mathrm{mg}^{-1}$ respectively.

The NrfA nitrite reductase system has been shown previously to play a role in detoxifying NO added exogenously to Salmonella and $E$. coli [26-30]. Some reports have suggested that a product of NO metabolism by $\mathrm{Nrf}$ can be $\mathrm{N}_{2} \mathrm{O}$ [31]. To assess this, a $n r f A$ periplasmic nitrite reductase mutant was also examined in nitraterich continuous culture, but, like the nirB mutant, it behaved identically with wild-type. Thus, although $n r f A$ was expressed in the anoxic phase of the cultures (Figure 2), the periplasmic NrfA enzyme does not appear to be important for detoxification of endogenously produced intracellular NO.

\section{Nitrate metabolism in nitrate-limited continuous cultures}

The net consumption of $\sim 17 \mathrm{mM}$ nitrate and $5 \mathrm{mM}$ glycerol by the nitrate-sufficient cultures suggested that running the continuous cultures with a $\mathrm{S}_{\mathrm{R}} \mathrm{NO}_{3}{ }^{-}$of $<17 \mathrm{mM}$ and a $\mathrm{S}_{\mathrm{R}}$ glycerol of $>5 \mathrm{mM}$ would lead to nitrate limitation. To achieve this condition, the $\mathrm{S}_{\mathrm{R}} \mathrm{NO}_{3}{ }^{-}$was lowered to $5.5 \mathrm{mM}$ and the $\mathrm{S}_{\mathrm{R}}$ glycerol concentration increased to $22 \mathrm{mM}$ (Figure 6A). Under these conditions, the $\mathrm{S}_{\mathrm{BR}} \mathrm{NO}_{3}{ }^{-}$was very low under steady-state conditions (100-120 h) (Figure 6B), consistent with nitrate limitation. qRT-PCR revealed up-regulation of nap, but not narG, following the oxic-anoxic transition, consistent with Nap being the enzyme of choice for metabolism under nitrate-limited 


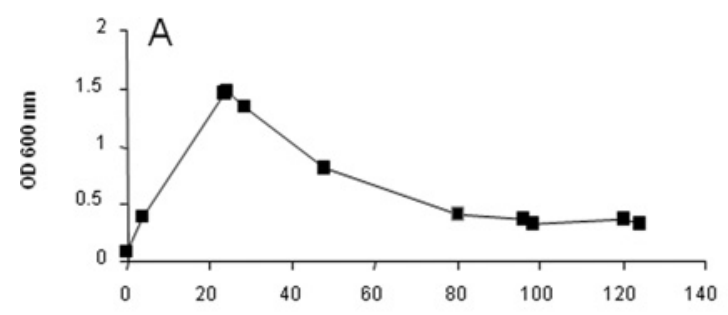

B

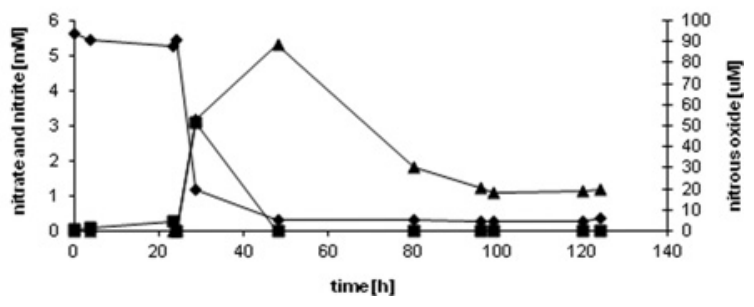

Figure 6 Nitrate, nitrite and $\mathrm{N}_{2} \mathrm{O}$ consumption or production in a glycerolsufficient nitrate-limited continuous culture of Salmonella Typhimurium SL1344

The culture was run as described for Figure 1. The glycerol concentration in the reservoir feed was $22 \mathrm{mM}$ and nitrate concentration was $5 \mathrm{mM}$. (A) biomass; (B) nitrate ( $)$, nitrite $(\mathbf{\square})$ and $\mathrm{N}_{2} \mathrm{O}(\boldsymbol{\Delta})$. Results are illustrative for experiments run in triplicate. For clarity, only the results from a single chemostat run are shown. The results ( \pm S.D.) derived from replicate experiments are given in Table 2.

conditions (Figure 2). During the transition phase of $24-48 \mathrm{~h}$, nitrite accumulated in the reactor vessel to a maximum of $\sim 3 \mathrm{mM}$, concomitantly with nitrate consumption. However, this was only transient, and nitrite was not detectable (detection limit of $0.005 \mathrm{mM}$ ) when the cultures reached steady state (Figure 6B). Thus the rates of nitrate and nitrite consumption (Reactions 1 and 2) were matched (Table 2). Significantly, the profile of $\mathrm{N}_{2} \mathrm{O}$ accumulation was quite different from the nitrate-sufficient cultures. Like nitrite, $\mathrm{N}_{2} \mathrm{O}$ accumulated transiently, and the peak of production lagged approximately $20 \mathrm{~h}$ behind that of nitrite, but the maximum obtained was 20 -fold lower than the maximum obtained in the nitrate-sufficient cultures (compare Figure 6B with Figure $1 \mathrm{~B})$. In steady state $(100-120 \mathrm{~h})$, there was only minimal $\mathrm{N}_{2} \mathrm{O}$ release, the rate of which was more than two orders of magnitude lower than for the nitrate-sufficient cultures (Figure 6B and Table 2), with less than $0.1 \%$ of the nitrate-nitrogen ending up as $\mathrm{N}_{2} \mathrm{O}$.

\section{DISCUSSION}

In the present study, we have examined nitrate catabolism and associated exogenous $\mathrm{N}_{2} \mathrm{O}$ production during continuous culture of Salmonella Typhimurium under nitrate-rich and nitrate-limited anoxic conditions. $\mathrm{NO}$ is detoxified by conversion into $\mathrm{N}_{2} \mathrm{O}$ in the cytoplasm (Reaction 4), and, as a consequence, the direct measurement of NO released by bacteria will grossly underestimate the actual level produced intracellularly during nitrate metabolism. Salmonella Typhimurium cannot reduce $\mathrm{N}_{2} \mathrm{O}$ and so measuring its extracellular release is a good quantitative measure for the fraction of nitrate catabolized that forms NO intracellularly. This has been illustrated in the present study where, under nitrate-rich conditions, millimolar levels of extracellular $\mathrm{N}_{2} \mathrm{O}$ were measured, but extracellular $\mathrm{NO}$ was not detected above $1 \mu \mathrm{M}$. This demonstrates that, under the steadystate metabolic conditions established, there is highly efficient reductive detoxification of endogenously produced cytotoxic NO to $\mathrm{N}_{2} \mathrm{O}$, which then escapes from the cell. From this, it can be estimated that up to $20 \%$ of nitrate catabolized is converted into the NO, which represents a substantial scale of intracellular production and thus requires very efficient detoxification of the cytotoxin.

$\mathrm{N}_{2} \mathrm{O}$ production was maximal under nitrate-rich culture conditions where both nar and nap were expressed. However, mutagenesis confirmed that Nar was the major enzymatic route for the nitrate catabolism associated with $\mathrm{N}_{2} \mathrm{O}$ production. (Figure 4 and Table 2). In the $20 \mathrm{~h}$ following anoxia, nitrate was consumed and nitrite accumulated in a near-stoichiometric fashion and very little $\mathrm{N}_{2} \mathrm{O}$ accumulated (Figure 1B). The likely biochemical processes in operation are first nitrate import via the nitrate/nitrite antiporter NarK [32], the gene for which was also up-regulated during nitrate catabolism under nitrate-sufficient conditions. This is then followed by reduction to nitrite by NarG, and export of nitrite by NarK in exchange for incoming nitrate (Figure 7A). This process generates protonmotive force for growth. It is notable that, under these nitrate-rich culture conditions, the consumption of the nitrite produced from nitrate respiration is minimal and there is extensive extracellular accumulation of nitrite rather than the further reduction to ammonium (Figure 1B) despite expression of both nirB and $n r f A$ (Figure 7A). Thus, although Salmonella Typhimurium is considered to be a canonical DNRA organism, under these nitraterich conditions, nitrite, rather than ammonium, is the major extracellular end-product of nitrate respiration. It makes sense from a bioenergetic view point to maximize nitrate respiration and minimize nitrite respiration under electron acceptor-sufficient conditions. This is because the $\uparrow \mathrm{H}^{+} / \mathrm{e}^{-}$stoichiometry for reduction of nitrite by NrfA ( 2 with $\mathrm{NADH}$ as electron donor and 0 with quinol as electron donor) is lower than for nitrate reduction by $\mathrm{Nar}$ (3 with $\mathrm{NADH}$ and 1 with quinol) [33]. In this respect, it also makes bioenergetic sense to utilize the NarG nitrate reductase system, rather than the Nap system, since the $\uparrow \mathrm{H}^{+} / \mathrm{e}^{-}$coupling ratios for periplasmic reduction of nitrate by Nap ( 2 with NADH and 0 with quinol) are also less than for Nar. This is highlighted by the lower biomass yield when the mutant in $n \operatorname{ar} G$, that is dependent on Nap for growth, was cultured under the nitrate-sufficient conditions (Table 2).

At the end of the transition phase of the anoxic nitrate-sufficient continuous culture, the nitrite reaches an extracellular level that is $\sim 3$-fold higher than that of nitrate (Figure 1). It is under these conditions that the rate of $\mathrm{N}_{2} \mathrm{O}$ production is maximal. The rate of $\mathrm{N}_{2} \mathrm{O}$ production matched the rate of nitrite reduction and the levels produced accounted for the balance of nitrate-nitrogen that did not accumulate as nitrite-nitrogen. The very low rate of $\mathrm{N}_{2} \mathrm{O}$ production in the narG mutant suggested that it was linked to nitrite reduction by NarG. Competition between two substrates (nitrite and nitrate) for a single active site can be described by:

$$
\begin{aligned}
v_{\text {nitrite }} / v_{\text {nitrate }}= & \left(k_{\text {cat }} / K_{\mathrm{m}}\right)_{\text {nitrite }} /\left(k_{\text {cat }} / K_{\mathrm{m}}\right)_{\text {nitrate }} \\
& \times([\text { nitrite }] /[\text { nitrate }])(\text { adapted from [34] })
\end{aligned}
$$

From the steady-state chemostat fluxes (Table 2), $v_{\text {nitrite }} / v_{\text {nitrate }}=\mathrm{qcNO}_{2}{ }^{-} / \mathrm{qcNO}_{3}{ }^{-}=0.226$. From the kinetic parameters derived from the purified NarGH, $\left(k_{\text {cat }} / K_{\mathrm{m}}\right)_{\text {nitrite }} /\left(k_{\text {cat }} /\right.$ $\left.K_{\mathrm{m}}\right)_{\text {nitrate }}=0.007$. Thus [nitrite] $/[$ nitrate $]=32$ and this equates to the steady-state intracellular ratio of the two substrates. Such a ratio is perfectly conceivable if some of the nitrite exported by NarK in exchange for incoming nitrate re-enters the cell, possibly via by the bidirectional nitrite channel NirC [31], the gene for which was expressed under these growth conditions (Figures 2 
A. High nitrate : nitrite and carbon-limitation

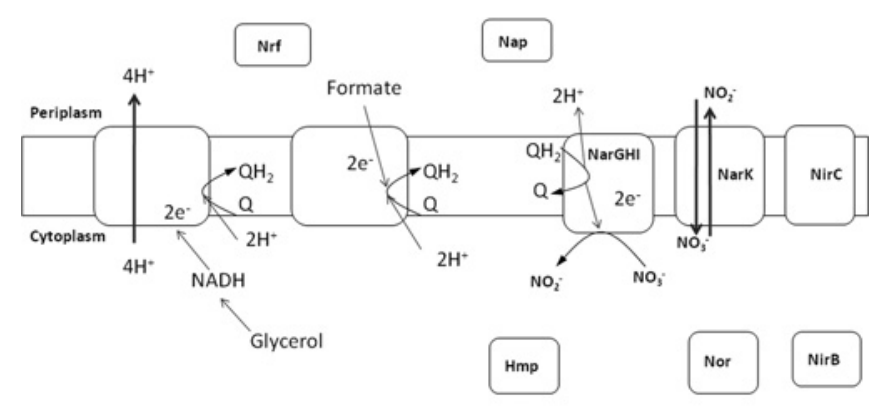

B. Low nitrate : nitrite and carbon-limitation

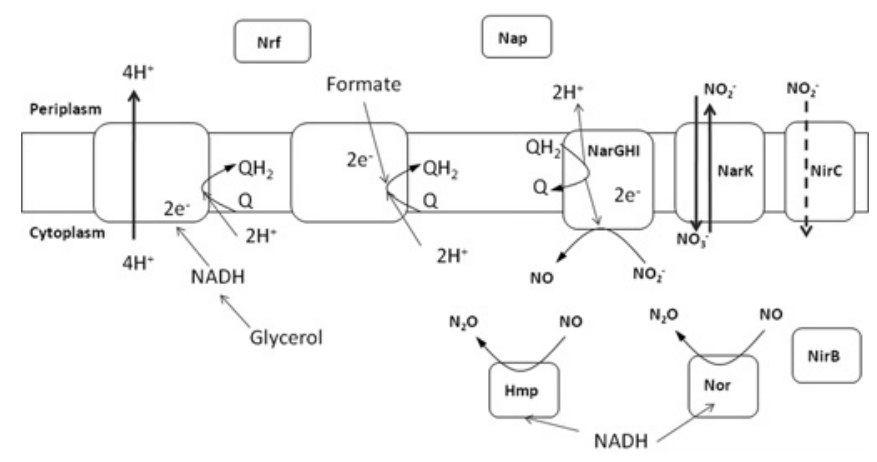

C. Nitrate-limited and carbon-sufficient

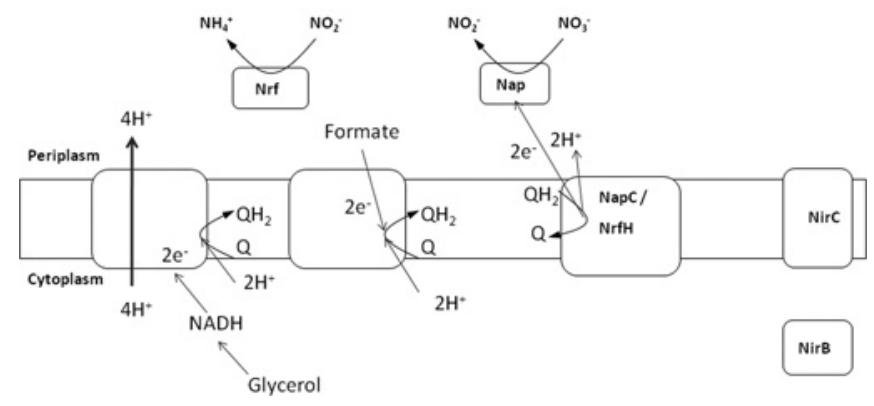

Figure 7 Schemes for nitrate and nitrite metabolism in Salmonella Typhimurium SL1344 under the different growth conditions explored in the present study

(A) In electron-acceptor-rich high-nitrate/nitrite conditions. Nar reduces nitrate to nitrite which is exported via Nark to the periplasm where it accumulates almost stoichiometrically with the nitrate consumed. (B) In electron-acceptor-rich low-nitrate/nitrate ratios, nitrite is imported by NirC and consumed via Nar producing NO, which is detoxified by Hmp and NorV to produce $\mathrm{N}_{2} \mathrm{O}$. (C) Under nitrate-limiting growth conditions, Nar is not synthesized, and the Nap and Nrf systems are actively consuming nitrate and the nitrite produced from nitrate reduction (with electrons flowing via the $\mathrm{NapC}$ and $\mathrm{NrfH}$ quinol dehydrogenases). No intracellular NO is generated, hmp and norV expression is low and $\mathrm{N}_{2} \mathrm{O}$ is not produced.

and 7B). Intracellular nitrite may then out-compete intracellular nitrate for the active site of a NarG. This argument is supported by the experiments with the NarG mutant under nitrate-sufficient conditions that showed a near-stoichiometric consumption of nitrate and production of nitrite, and a very low level of $\mathrm{N}_{2} \mathrm{O}$ production in the steady state (Figure 4). It is also consistent with the data of the early 1980 s that led to the conclusion that NarG might reduce nitrite to $\mathrm{N}_{2} \mathrm{O}$ and with more recent genetic and microbiological data that suggested that NarG in fact reduces nitrite to NO $[3,11,12,18-20]$.
Under nitrate-limited growth conditions, the production of $\mathrm{N}_{2} \mathrm{O}$ was very low in the steady-state phase, where both nitrate and nitrite were only present at low-micromolar levels. These conditions promoted expression of napA and $n r f A$, but not narG (Figure 2). This is consistent with the expression pattern observed in $E$. coli under low-nitrate/nitrite growth conditions [22,23,35-37]. Under these conditions, the absence of nitrite accumulation reflects that it is fully reduced through to ammonium by NrfA (Figure 7C, Reaction 2), so that there is maximum utilization of the limited respiratory electron-acceptor pool available to the culture. Thus results of the present study for Salmonella Typhimurium confirm observations from $E$. coli that when nitrate is sufficiently abundant, the bacteria exploit the energy-efficient, but low-affinity, NarG enzyme to reduce nitrate in the cytoplasm [22,23,35-37]. When nitrate is scarce, Nap provides a higher-affinity, but more poorly coupled, pathway that does not require nitrate transport for nitrate to serve as an effective electron sink [21] (Figure 7C).

Broadening the implications of the results more widely for the many species of Enterobacteriaceae that synthesize both NarG and NapA, the primary role of NarG is to generate protonmotive force when nitrate is abundant, such as occurs in nitrate-rich carbon-limited soils and sediments or wastewater-treatment plants [2]. Under these conditions, it has previously been thought that nitrite reductase NirB protects the cytoplasm from nitrite toxicity. However, the results of the present study show that nitrite can accumulate to millimolar levels in the extracellular medium and that, in fact, what nitrite is consumed is reduced to $\mathrm{N}_{2} \mathrm{O}$ (Reactions 3 and 4) not ammonium (Reaction 2). However, in many habitats, Enterobacteriacaea will encounter much lower concentrations of nitrate, where the periplasmic pathway for nitrate and nitrite reduction is active [2]. We have shown that this combination will not lead to intracellular NO production, as judged by the lack of $\mathrm{N}_{2} \mathrm{O}$ production and the absence of up-regulation of the $h m p$ and $h c p$ genes of NsrR regulon. The comparative experiments conducted with the narG and napA mutants demonstrate that nitrate reduction by Nap does not lead to extensive $\mathrm{N}_{2} \mathrm{O}$ production even under nitrate-sufficient conditions.

Comparison of the narG and napA strains revealed a higher cell yield using Nar rather than Nap, reflecting the higher $\uparrow \mathrm{H}^{+} / \mathrm{e}^{-}$for quinol oxidation by nitrate for Nar (1) compared with Nap (0) [33]. When Nap is operational, growth is dependent on energyconserving formate dehydrogenase and NADH dehydrogenase reactions associated with glycerol metabolism for energy conservation, with nap serving a quinol pool recycling role [33]. However, although it makes bioenergetic sense to use NarG, under nitrate-rich conditions, there is a downside, which is the risk of cell damage associated with cytotoxic NO production. Coordinate induction of a NO-detoxification system minimizes this risk. There is, however, an energetic cost in using electrons in the non-energy-conserving cytoplasmic reduction of NO. The overall reduction of nitrite to $\mathrm{N}_{2} \mathrm{O}$ via $\mathrm{NarG}$ and then $\mathrm{HmpA}$ or NorV using NADH consumes 2 mol of NADH (4 mol of $\mathrm{e}^{-}$; Reactions 3 and 4 ) and yields an overall $\uparrow \mathrm{H}^{+} / \mathrm{e}^{-}$of 1.5 , which compares with an overall $\uparrow \mathrm{H}^{+} / \mathrm{e}^{-}$of 2 when $\mathrm{NADH}$ is used via the respiratory electron-transport chain to reduce nitrite to ammonium. Thus it merits reflection on whether the diversion of $\sim 20 \%$ of nitrate into $\mathrm{NO}$ and thence $\mathrm{N}_{2} \mathrm{O}$ has any physiological importance. $\mathrm{N}_{2} \mathrm{O}$ is a neuropharmacological agent that inhibits a range of cell receptors and transporters. The significance of $\mathrm{N}_{2} \mathrm{O}$ production by pathogens has not been addressed, but it is likely to be a property of many enterobacterial pathogens and also pathogens such as Neisseria that have truncated denitrification pathways in which $\mathrm{N}_{2} \mathrm{O}$ is the product of nitrite reduction due to the lack of $\mathrm{N}_{2} \mathrm{O}$ reductase [38,39]. Further studies on the significance of $\mathrm{N}_{2} \mathrm{O}$ production by pathogenic bacteria are therefore merited. 


\section{AUTHOR CONTRIBUTION}

Daniela Hensen designed and executed the continuous culture experiments. Heather Felgate and Anke Arkenberg contributed to the continuous culture experiments. Corinne Appia-Ayme undertook the qRT-PCR experiments. Karen Prior and Carl Harrington constructed some of the mutants. Sarah Field undertook the enzyme kinetics. Julea Butt and Elizabeth Baggs contributed to the experimental concepts and discussion of the results. Gary Rowley and David Richardson designed the experiments and wrote the paper.

\section{ACKNOWLEDGEMENTS}

We thank Lucy Cousins for excellent technical support.

\section{FUNDING}

This work was supported by the Biotechnology and Biological Sciences Research Council [grant numbers BB/D012384/1 and BB/D010942/1] and a Biotechnology and Biological Sciences Research Council Ph.D. studentship to H.F. D.J.R. thanks the Royal Society and Wolfson Foundation for a Merit Award. We thank the Royal Society and the Wolfson Foundation for providing capital funds for the Wolfson Bionergy and Fermentation Laboratory at the University of East Anglia.

\section{REFERENCES}

1 Cole, J. (1996) Nitrate reduction to ammonia by enteric bacteria: redundancy, or a strategy for survival during oxygen starvation? FEMS Microbiol. Lett. 136, 1-11

2 Potter, L., Angove, H., Richardson, D. and Cole, J. (2001) Nitrate reduction in the periplasm of Gram-negative bacteria. Adv. Microb. Physiol. 45, 51-112

3 Gilberthorpe, N. J. and Poole, R. K. (2008) Nitric oxide homeostasis in Salmonella Typhimurium: roles of respiratory nitrate reductase and flavohemoglobin. J. Biol. Chem. 283, 11146-11154

4 Spiro, S. (2006) Nitric oxide-sensing mechanisms in Escherichia coli. Biochem. Soc Trans. 34, 200-202

5 Gilberthorpe, N. J., Lee, M. E., Stevanin, T. M., Read, R. C. and Poole, R. K. (2007) NsrR: a key regulator circumventing Salmonella enterica serovar Typhimurium oxidative and nitrosative stress in vitro and in IFN- $\gamma$-stimulated J774.2 macrophages. Microbiology 153, 1756-1771

6 Bodenmiller, D. M. and Spiro, S. (2006) The yjeB (nsrR) gene of Escherichia coli encodes a nitric oxide-sensitive transcriptional regulator. J. Bacteriol. 188, 874-881

7 Filenko, N., Spiro, S., Browning, D. F., Squire, D., Overton, T. W., Cole, J. and Constantinidou, C. (2007) The NsrR regulon of Escherichia coli K-12 includes genes encoding the hybrid cluster protein and the periplasmic, respiratory nitrite reductase. J. Bacteriol. 189, 4410-4417

8 Spiro, S. (2007) Regulators of bacterial responses to nitric oxide. FEMS Microbiol. Rev. 31, 193-211

9 Kim, S. O., Orii, Y., Lloyd, D., Hughes, M. N. and Poole, R. K. (1999) Anoxic function for the Escherichia coli flavohaemoglobin (Hmp): reversible binding of nitric oxide and reduction to nitrous oxide. FEBS Lett. 445, 389-394

10 Richardson, D., Felgate, H., Watmough, N., Thomson, A. and Baggs, E. (2009) Mitigating release of the potent greenhouse gas $\mathrm{N}_{2} \mathrm{O}$ from the nitrogen cycle: could enzymic regulation hold the key? Trends Biotechnol. 27, 388-397

11 Bleakley, B. H. and Tiedje, J. M. (1982) Nitrous oxide production by organisms other than nitrifiers or denitrifiers. Appl. Environ. Microbiol. 44, 1342-1348

12 Smith, M. S. (1983) Nitrous oxide production by Escherichia coli is correlated with nitrate reductase activity. Appl. Environ. Microbiol. 45, 1545-1547

13 Pope, N. R. and Cole, J. A. (1982) Generation of a membrane potential by one of two independent pathways for nitrite reduction by Escherichia coli. J. Gen. Microbiol. 128, 219-222

14 Smith, P. K., Krohn, R. I., Hermanson, G. T., Mallia, A. K., Gartner, F. H., Provenzano, M. D., Fujimoto, E. K., Goeke, N. M., Olson, B. J. and Klenk, D. C. (1985) Measurement of protein using bicinchoninic acid. Anal. Biochem. 150, 76-85

15 Nicholas, D.J.D. and Nason, A. (1957), Determination of Nitrate and Nitrite, Academic Press, New York

16 Anderson, L. J., Richardson, D. J. and Butt, J. N. (2001) Catalytic protein film voltammetry from a respiratory nitrate reductase provides evidence for complex electrochemical modulation of enzyme activity. Biochemistry 40, 11294-11307
17 Datsenko, K. A. and Wanner, B. L. (2000) One-step inactivation of chromosomal genes in Escherichia coli K-12 using PCR products. Proc. Natl. Acad. Sci. U.S.A. 97, 6640-6645

18 Metheringham, R. and Cole, J. A. (1997) A reassessment of the genetic determinants, the effect of growth conditions and the availability of an electron donor on the nitrosating activity of Escherichia coli K-12. Microbiology 143, 2647-2656

$19 \mathrm{Ji}, \mathrm{X}$. B. and Hollocher, T. C. (1988) Reduction of nitrite to nitric oxide by enteric bacteria. Biochem. Biophys. Res. Commun. 157, 106-108

20 Satoh, T., Hom, S. S. and Shanmugam, K. T. (1983) Production of nitrous oxide from nitrite in Klebsiella pneumoniae: mutants altered in nitrogen metabolism. J. Bacteriol. 155, 454-458

21 Potter, L. C., Millington, P., Griffiths, L., Thomas, G. H. and Cole, J. A. (1999) Competition between Escherichia coli strains expressing either a periplasmic or a membrane-bound nitrate reductase: does Nap confer a selective advantage during nitrate-limited growth? Biochem. J. 344, 77-84

22 Stewart, V., Bledsoe, P. J. and Williams, S. B. (2003) Dual overlapping promoters control napF (periplasmic nitrate reductase) operon expression in Escherichia coli K-12. J. Bacteriol. 185, 5862-5870

23 Wang, H., Tseng, C. P. and Gunsalus, R. P. (1999) The napF and narG nitrate reductase operons in Escherichia coli are differentially expressed in response to submicromolar concentrations of nitrate but not nitrite. J. Bacteriol. 181, 5303-5308

24 van Riet, J., van Ed, J. H., Wever, R., van Gelder, B. F. and Planta, R. J. (1975) Characterization of the respiratory nitrate reductase of Klebsiella aerogenes as a molybdenum-containing iron-sulfur enzyme. Biochim. Biophys. Acta 405, 306-317

25 Weiss, B. (2006) Evidence for mutagenesis by nitric oxide during nitrate metabolism in Escherichia coli. J. Bacteriol. 188, 829-833

26 Burlat, B., Gwyer, J. D., Poock, S., Clarke, T., Cole, J. A., Hemmings, A. M., Cheesman, M. R., Butt, J. N. and Richardson, D. J. (2005) Cytochrome $c$ nitrite reductase: from structural to physicochemical analysis. Biochem. Soc. Trans. 33, 137-140

27 Clarke, T. A., Mills, P. C., Poock, S. R., Butt, J. N., Cheesman, M. R., Cole, J. A., Hinton, J. C., Hemmings, A. M., Kemp, G., Soderberg, C. A. et al. (2008) Escherichia coli cytochrome $c$ nitrite reductase NrfA. Methods Enzymol. 437, 63-77

28 Poock, S. R., Leach, E. R., Moir, J. W., Cole, J. A. and Richardson, D. J. (2002) Respiratory detoxification of nitric oxide by the cytochrome $c$ nitrite reductase of Escherichia coli. J. Biol. Chem. 277, 23664-23669

29 Mills, P. C., Rowley, G., Spiro, S., Hinton, J. C. and Richardson, D. J. (2008) A combination of cytochrome $c$ nitrite reductase (NrfA) and flavorubredoxin (NorV) protects Salmonella enterica serovar Typhimurium against killing by NO in anoxic environments. Microbiology 154, 1218-1228

30 van Wonderen, J. H., Burlat, B., Richardson, D. J., Cheesman, M. R. and Butt, J. N. (2008) The nitric oxide reductase activity of cytochrome $c$ nitrite reductase from Escherichia coli. J. Biol. Chem. 283, 9587-9594

31 Costa, C., Macedo, A., Moura, I., Moura, J. J., Le Gall, J., Berlier, Y., Liu, M. Y. and Payne, W. J. (1990) Regulation of the hexaheme nitrite/nitric oxide reductase of Desulfovibrio desulfuricans, Wolinella succinogenes and Escherichia coli: a mass spectrometric study. FEBS Lett. 276, 67-70

32 Jia, W., Tovell, N., Clegg, S., Trimmer, M. and Cole, J. (2009) A single channel for nitrate uptake, nitrite export and nitrite uptake by Escherichia coli NarU and a role for NirC in nitrite export and uptake. Biochem. J. 417, 297-304

33 Simon, J., van Spanning, R. J. and Richardson, D. J. (2008) The organisation of proton motive and non-proton motive redox loops in prokaryotic respiratory systems. Biochim. Biophys. Acta 1777, 1480-1490

34 Fersht A (1985) Enzyme Structure and Mechanism, W.H. Freeman, San Francisco

35 Stewart, V., Bledsoe, P. J., Chen, L. L. and Cai, A. (2009) Catabolite repression control of napF (periplasmic nitrate reductase) operon expression in Escherichia coli K-12. J. Bacteriol 191, 996-1005

36 Stewart, V., Lu, Y. and Darwin, A. J. (2002) Periplasmic nitrate reductase (NapABC enzyme) supports anaerobic respiration by Escherichia coli K-12. J. Bacteriol. 184 1314-1323

37 Stewart, V. and Bledsoe, P. J. (2005) Fnr-, NarP- and NarL-dependent regulation of transcription initiation from the Haemophilus influenzae Rd napF (periplasmic nitrate reductase) promoter in Escherichia coli K-12. J. Bacteriol. 187, 6928-6935

38 Mellies, J., Jose, J. and Meyer, T. F. (1997) The Neisseria gonorrhoeae gene aniA encodes an inducible nitrite reductase. Mol. Gen. Genet. 256, 525-532

39 Barth, K. R., Isabella, V. M. and Clark, V. L. (2009) Biochemical and genomic analysis of the denitrification pathway within the genus Neisseria. Microbiology 155, 4093-4103

40 Hoiseth, S. K. and Stocker, B. A. (1981) Aromatic-dependent Salmonella Typhimurium are non-virulent and effective as live vaccines. Nature 291, 238-239

Received 1 June 2011/1 November 2011; accepted 1 November 2011

Published as BJ Immediate Publication 1 November 2011, doi:10.1042/BJ20110971 